Accepted on Neuropsychologia, December 2012

\title{
Context and Hand Posture Modulate the Neural Dynamics of Tool- Object Perception
}

Nikhilesh Natraj ${ }^{1}$, Victoria Poole ${ }^{2}$, J.C. Mizelle ${ }^{1,3}$, Andrea Flumini ${ }^{4}$, Anna M. Borghi ${ }^{4,5}$, Lewis A. Wheaton ${ }^{1}$

1 Cognitive Motor Control Laboratory, School of Applied Physiology, Georgia Tech, Atlanta, GA

2 Weldon School of Biomedical Engineering, Purdue University, West Lafayette, Indiana

3 Rehabilitation Research and Development, Atlanta Veterans Affairs Medical Center, GA

4 Department of Psychology, Bologna, Italy

$\underline{5 \text { Institute of Cognitive Sciences and Technologies, National Research Council, Rome, Italy }}$

Contact information

Lewis Wheaton, Ph.D.

School of Applied Physiology

Georgia Institute of Technology

555 14th Street, Room 1309E

Atlanta, GA 30332-0356

lewis.wheaton@ap.gatech.edu

Phone: 404-385-2339; Fax: 404-894-9982

and

Anna M. Borghi, Department of Psychology,

Viale Berti Pichat 5, 40127 Bologna, Italy

Email: annamaria.borghi@unibo.it

Phone: +39-051-2091838, Fax: +39-051-243086 
Abstract

Prior research has linked visual perception of tools with plausible motor strategies. Thus, observing a tool activates the putative action-stream, including the left posterior parietal cortex. Observing a hand functionally grasping a tool involves the inferior frontal cortex. However, tooluse movements are performed in a contextual and grasp specific manner, rather than relative isolation. Our prior behavioral data has demonstrated that the context of tool-use (by pairing the tool with different objects) and varying hand grasp postures of the tool can interact to modulate subjects' reaction times while evaluating tool-object content. Specifically, perceptual judgment was delayed in the evaluation of functional tool-object pairings (Correct context) when the tool was non-functionally (Manipulative) grasped. Here, we hypothesized that this behavioral interference seen with the Manipulative posture would be due to increased and extended left parietofrontal activity possibly underlying motor simulations when resolving action conflict due to this particular grasp at time scales relevant to the behavioral data. Further, we hypothesized that this neural effect will be restricted to the Correct tool-object context wherein action affordances are at a maximum.

64-channel electroencephalography (EEG) was recorded from 16 right-handed subjects while viewing images depicting three classes of tool-object contexts: functionally Correct (e.g. coffee pot - coffee mug), functionally Incorrect (e.g. coffee pot - marker) and Spatial (coffee pot - milk). The Spatial context pairs a tool and object that would not functionally match, but may commonly appear in the same scene. These three contexts were modified by hand interaction: No Hand, Static Hand near the tool, Functional Hand posture and Manipulative Hand posture. The Manipulative posture is convenient for relocating a tool but does not afford a functional engagement of the tool on the target object. Subjects were instructed to visually assess whether the pictures displayed correct tool-object associations. EEG data was analyzed in time-voltage and time-frequency domains. Overall, Static Hand, Functional and Manipulative postures cause 
early activation (100-400ms post image onset) of parietofrontal areas, to varying intensity in each context, when compared to the No Hand control condition. However, when context is Correct, only the Manipulative Posture significantly induces extended neural responses, predominantly over right parietal and right frontal areas [400-600ms post image onset]. Significant power increase was observed in the theta band $[4-8 \mathrm{~Hz}]$ over the right frontal area, [0$500 \mathrm{~ms}]$. In addition, when context is Spatial, Manipulative posture alone significantly induces extended neural responses, over bilateral parietofrontal and left motor areas [400-600ms]. Significant power decrease occurred primarily in beta bands [12-16, 20-25Hz] over the aforementioned brain areas [400-600ms].

Here, we demonstrate that the neural processing of tool-object perception is sensitive to several factors. While both Functional and Manipulative postures in Correct context engage predominantly an early left parietofrontal circuit, the Manipulative posture alone extends the neural response and transitions to a late right parietofrontal network. This suggests engagement of a right neural system to evaluate action affordances when hand posture does not support action (Manipulative). Additionally, when tool-use context is ambiguous (Spatial context), there is increased bilateral parietofrontal activation and, extended neural response for the Manipulative posture. These results point to the existence of other networks evaluating toolobject associations when motoric affordances are not readily apparent and underlie corresponding delayed perceptual judgment in our prior behavioral data wherein Manipulative postures had exclusively interfered in judging tool-object content.

\section{$\underline{\text { Keywords }}$}

Action Perception; Tool Use; Mirror Neuron; EEG; Affordances 


\section{Introduction}

Research has well shown that tool knowledge is a part of our neural architecture. Previous work has identified class-specific mechanisms of object recognition in the brain (Weisberg, van Turennout, \& Martin, 2007), and it is known that viewing tools activates inferior regions of the left intraparietal sulcus and ventral premotor cortex (L. L. Chao, Weisberg, \& Martin, 2002). Extensive tool-specific activation is also commonly seen at diffuse temporal regions (Beauchamp \& Martin, 2007). Although commonly active in response to tool stimuli, there does appear to be specialization in the type of information processed at these regions. Posterior parietal and premotor activation in response to tools may be specialized to convey information related to the motor affordance of a tool rather than simply its identity (Jeannerod, Arbib, Rizzolatti, \& Sakata, 1995; S. H. Johnson-Frey, 2004). Posterior and inferior temporal activation, however, seems to be of particular importance in tool identification rather than understanding motoric qualities (Martin, 2007). As such, specialized mechanisms seem to link the identification of manipulable objects with information about the actions associated with their use throughout distributed brain areas, an idea that is well supported by previous literature (Grezes \& Decety, 2002; Mahon et al., 2007).

However, tool-use movements are often not performed in isolation, but are reliant on specific context-use and grasp-use knowledge. Previous neuroimaging studies have shed light on the neural substrates underlying perceptual judgment of contextual relationships between tools and objects (Mizelle \& Wheaton, 2010b, 2010c). However, little is known on how the combination of tool-grasp and tool-use contexts is neurally encoded. For example, to eat soup out of a bowl, we must be able to recognize the advantage of using a spoon with the cup instead of a key (context-use), and further understand that the spoon is to be grasped by the stem rather than the bowl (grasp-use). Such knowledge may be formed by our prior experiences with these tools, allowing for the differentiation of utility (spoon versus key for use with the soup bowl) and proper grasp (stem versus bowl). In this case, tool-use must involve understanding not only proper tool-object interactions, but also proper grasping postures to use tools (Jacquet, Chambon, Borghi, \& Tessari, 2012). For the purposes of this work, we define a "tool" as an object used by an actor to act on something, which in this case is an "object". Further, we can interact with tools using different basic postures. "Functional" grasp identifies a grasp for the purposes of using the tool, while "Manipulative" grasp is not convenient for engaging in functional use but may be convenient for other actions, such as relocating the tool. For a similar distinction, we point to (Bub, Masson, \& Cree, 2008; Costantini, Ambrosini, Scorolli, \& Borghi, 2011; Pellicano, lani, Borghi, Rubichi, \& Nicoletti, 2010).

We extended our conceptual relationships findings of previous studies (Mizelle \& Wheaton, 2010b, 2010c) with a behavioral paradigm focusing on interference of grasp postures on judgment. The behavioral paradigm (Borghi, Flumini, Natraj, \& Wheaton, 2012) revealed that perceptual judgment of tool-use is affected by the context of tool-object relationships and the hand posture of the tool grasp. We had focused on three levels of tool object relationships: Correct (e.g., coffee pot - coffee cup), Incorrect (e.g., coffee pot - marker) and Spatial (e.g., coffee pot - milk). The Spatial context was based on two items that are commonly together, but do not work together to create a tool-object action. These three levels were then modified based on hand interaction: No Hand, Static Hand (at the bottom of the picture roughly equidistant from tool and object), Functional posture of hand, and Manipulative posture of the 
hand. Specifically, we found that Manipulative postures elongated the decision process of judging if tool-object relationships were correct, compared to other hand postures (Functional, Static Hand or No Hand). Importantly, this effect was most pronounced when the tool object context was Correct. The Spatial tool object context always elicited the longest judgment times and within this context, the Manipulative and Functional postures were slower than No Hand, with the Manipulative posture eliciting the longest judgment times. Thus, Manipulative postures exclusively interfered with the decision about tool-object usage. Our goal in this current study was to identify the neural architecture underlying the results from the behavioral study, notably assessing parietofrontal circuit activity (with electroencephalography, EEG) at time scales relevant to the behavioral data. Our overall hypothesis was that the behavioral interference seen with the Manipulative posture would be due to increased and extended event related potential activity over left parietofrontal areas known to be involved in tool-use knowledge and action encoding (L. Chao \& Martin, 2000; S. Johnson-Frey, Newman-Norlund, \& Grafton, 2005; S. H. Johnson-Frey, 2004; Kroliczak \& Frey, 2009). This may relate to underlying motor simulations to resolve tool-object action interfered by the manipulative grasp. Further, we hypothesized that the increased parietofrontal activation would be limited to the Correct context wherein action affordances are at a maximum. Other contexts (Incorrect and Spatial) serve to evaluate if hand postures affect action encoding over left parietofrontal areas when the tools and objects do not afford mutual action themselves. Specifically, we hypothesized that unique to the Correct context, a) Manipulative postures would uniquely evoke stronger left parieto-frontal-motor activation compared to the No Hand condition with corresponding beta band spectral differences indicating motoric processing of the stimulus (Mizelle, Tang, Pirouz, \& Wheaton, 2011; Van Elk, Van Schie, Van Den Heuvel, \& Bekkering, 2010; L. A. Wheaton, Nolte, Bohlhalter, Fridman, \& Hallett, 2005) and b) Manipulative posture activations would be prolonged relative to the comparisons of No Hand versus Static Hand, or No Hand versus Functional posture, with the latter two showing differences earlier in time indicative of more rapid processing to tool-use. Our previous work suggests this prolonged response may be due to an interference effect of the Manipulative posture (Borghi, et al., 2012), possibly underlying action decoding.

\section{Materials and Methods}

\subsection{Subjects}

Sixteen (16) right-handed adult subjects (8 male, mean age, 21.2; SD, 1.3) were recruited for this research study. The experimental protocol was approved by the Institutional Review Board at Georgia Institute of Technology and each subject provided their written informed consent before the start of the experimental session. Subjects were healthy based on self-report, and had no history of neurological illness or injury.

\subsection{Visual Stimuli}

We used the same stimuli from our previous study (Borghi, et al., 2012) which were images composed of tools, objects, and a hand interacting with the tools in various grasps. In this prior study, all images were normed according to visual complexity, tool recognition, and familiarity. There were 3 possible tool use contexts within 4 possible hand postures, creating 12 conditions for any one tool ( 3 contexts by 4 hand postures). Figure 1 gives a depiction of all conditions for a particular tool. 
The context of tool usage was determined by its pairing with another object. For example, forkstrawberries would be a Correct tool-object pairing, fork-tennis ball would be an Incorrect tool object pairing, fork-glass would be a Spatial tool object pairing. In these tool-object scenes, there were four hand variations. The hand was either absent (No Hand), reaching into the scene but not touching the tool (Static Hand), holding the handle (Functional), or holding the opposite end of the tool (Manipulative).

\subsection{Data Collection}

Subjects were seated comfortably in a chair with no restraints and fitted with a standard 64 channel tin electrode EEG cap (Electrocap, Eaton, $\mathrm{OH}$ ) in accordance with the international 1020 system. Neural activity was recorded using Synamps 2 (Neuroscan, Charlotte, NC). As well, we placed two electrodes above and below the left eye to record electrooculographic activity (EOG). These EOG channels were used offline to extract ocular artifacts (eye movement and eyeblink) from the EEG signal using adaptive filtering. Data acquisition was performed using a right ear reference at a sampling rate of $1000 \mathrm{~Hz}$. The left ear was also recorded and was used offline in creating a linked ears reference. Visual stimuli were presented on a 20 inch widescreen display using Stim (Neuroscan, Charlotte, NC), at a distance of 6 feet from the subjects. The display was placed in the middle of their visual field and the height of the display was matched to the seated eye of the subject.

\subsection{Experimental Paradigm}

For this study, we used 23 tools (Borghi, et al., 2012) with 12 possible variants of grasp and context within each tool (12 conditions); therefore there were a total of $23 \times 12=276$ images. Sorting all the images based on condition gave 23 images per condition. For example, the condition 'Spatial context, Functional grasp' consisted of 23 images, one per tool. Each image was presented twice. Due to the large number of potential images, 17 (out of 23) images per condition were pseudo-randomly pre-selected for each subject. Therefore each subject viewed 408 images in total (17 pictures X 12 conditions $X 2$ presentations per picture). The presentation of these visual stimuli was equally distributed across 4 data acquisition blocks. Each block lasted approximately 11 minutes. The design of each block is outlined in Figure 2.

Subjects viewed two sequential cues followed by the stimulus. The first cue was a circle, lasting $4000 \mathrm{~ms}$, to indicate a rest phase. The second cue was a cross lasting $500 \mathrm{~ms}$, which indicated an image was pending. Immediately following the cross, the target tool-object image was presented on the screen for $2000 \mathrm{~ms}$. The circle cue then reappeared, and this cycle of restwarning-stimulus repeated for the duration of the block. Continuous EEG was recorded for this duration. Image presentations were synched with the continuous EEG traces using Stim2 (Compumedics, Charlotte, NC). Unique codes generated by Stim2 identified the onset of each image presentation in the EEG signal to facilitate analysis. Subjects were instructed to judge the appropriateness of the tool-object content in the image and passively decide if the scene was correct. Since behavioral analyses had already been performed in our prior work (Borghi, et al. 2012), we avoided a behavioral response (such as button press) so that motor control processing (motor execution/inhibition) would not interfere with the data interpretation. 


\subsection{Data Analysis}

The raw EEG data was first low pass filtered from $\mathrm{DC}-30 \mathrm{~Hz}$. An epoch was then constructed by extracting data from $1000 \mathrm{~ms}$ before to $2000 \mathrm{~ms}$ after tool-object image onset. Each epoch was therefore $3000 \mathrm{~ms}$ long $(-10002000 \mathrm{~ms})$, with 0 corresponding to appearance of the tool-object image on screen and -500 corresponding to presentation of the cross indicating that an image was pending (Figure 2). Using custom scripts in Neuroscan and MATLAB (Mathworks, MA), the extracted epochs were then linear detrended and baseline corrected to the first $500 \mathrm{~ms}(-1000$ to $-500 \mathrm{~ms}$ ), until onset of the warning cue. Next, the epochs were sorted based on the 12 conditions. Epochs were then corrected for ocular artifacts (eye movement and eye blink) using the Recursive Least Squares Algorithm (He, Wilson, \& Russell, 2004; Liavas \& Regalia, 1999) implemented through the EEGLAB AAR Toolbox (Delorme \& Makeig, 2004; Gómez-Herrero, 2007). The exponential weighting of the least squares error while calculating the adaptive filter coefficients makes RLS attractive for EEG artifact correction, as it follows the non-stationary trends of the EEG signal very well (Hayes, 2009). A threshold magnitude of $75 \mu \mathrm{V}$ was applied to reject epochs with residual noise. Research has shown that visually evoked response amplitudes are susceptible to non-task related differences, such as gender, societal influences (Steffensen et al., 2008) and age (Falkenstein, Hoormann, \& Hohnsbein, 2001). To counter this intersubject variance, data for all 16 subjects were resampled to 1000 epochs according to the bootstrap procedure (Efron, 1981; Mizelle \& Wheaton, 2010a). Sixteen (16) new pseudosubjects were created from the resampling procedure and these data were then subjected to time voltage analysis. Further analysis was performed in the time frequency domain.

\subsubsection{Time Voltage Analysis}

To assess regional voltage-based effects, five Regions of Interest (ROI) were constructed based on the 10-20 convention (Jasper, 1958): Left frontal: electrodes F7, F5, F3; Right frontal: electrodes F4, F6, F8; Left Motor: electrodes C3, C1, Cz; Left Parieto-temporal: electrodes TCP1, C3P, P5, P3; Right Parieto-temporal: electrodes TCP2, C4P, P4, P6.

Time voltage waveforms were grand averaged per subject and condition across each ROI to obtain visual evoked responses at the single subject level. Given the complexity of the stimuli that could elicit multiple peaks in the visual response with subtle variations from person to person, the visual evoked responses were windowed and mean voltages were analyzed (area under the evoked response). Therefore, based on the location and characteristics of these peaks, three windows of interest were chosen post hoc: Early phase (100-200 ms) after picture presentation; Middle phase (200-400 ms) after picture presentation; Late phase (400-600 ms) after picture presentation. Since our prior behavioral study had average subject response time of about $750 \mathrm{~ms}$ for the same visual stimuli, we did not analyze any EEG epoch beyond 600ms (with the $150 \mathrm{~ms}$ differential to account for the motoric processing underlying subject's response required in our prior work) (Borghi, et al., 2012). Analyzing binned data this way made it easier for us to relate our neural activations to time scales from our behavioral study. Within each time window, voltage values were averaged within subjects and conditions for each ROI to yield a mean voltage for each ROI, Subject and Condition. Statistical analyses were performed on the resultant means. For each time window, voltage means were compared using a 3-way analysis of variance (ANOVA) at the $\mathrm{p}<0.05$ level, with the following within-subject factors: Hand (4 levels; No Hand, Static Hand, Functional, Manipulative), Context (3 levels; Correct, Incorrect and Spatial), and Region (5 levels; Right and Left Frontal, Left Motor, Left and Right Parieto- 
Temporal). Post-hoc t-tests were used to determine effects of Hand within each $\mathrm{ROI}(\mathrm{p}<0.01)$, wherever allowable. As our interests were focused on the role of hand postures within a context, the No Hand condition was used as control and individual t-tests were run (Static Hand vs. No Hand, Functional vs. No Hand, Manipulative vs. No Hand) at each ROI, within each context to estimate the effect of hand posture on evoked responses. In line with our hypotheses, the posthoc t-tests design did not compare all hand and context conditions with each other directly (such as Manipulative vs. Functional) (Nieuwenhuis, Forstmann, \& Wagenmakers, 2011), since we were not interested in the differences between a Functional and Manipulative grasp per se. Our primary interests were in contrasting the Manipulative posture vs. the No Hand control condition within each context to infer unique spatiotemporal activations due to this hand posture that might speak to our prior behavioral results. As well, we aimed to examine if similar differences against control exist for the Functional and Static Hand postures. To this end post-hoc tests were carried out relative to the No Hand condition and we discuss the spatiotemporal differences in post-hoc contrasts (Functional vs. No Hand, Static Hand vs. No Hand within respective contexts).

\subsubsection{Time Frequency Analysis:}

We evaluated the time varying properties of the EEG signal in the frequency domain. We carried out time frequency analyses in Matlab using EEGLAB (Delorme \& Makeig, 2004), specifically evaluating Event Related Spectral Perturbations (ERSP) (Makeig, 1993; Makeig. Debener, Onton, \& Delorme, 2004). ERSP are generalizations of ERD/ERS (Event Related De/Synchronization) in the power spectra (Makeig, et al., 2004; Pfurtscheller \& Lopes da Silva, 1999). An increase in power with respect to baseline is representative of ERS whereas a loss in power with respect to baseline is characterized as ERD. ERD/ERS patterns indicate oscillatory activity of the underlying pool of neurons in response to task demands. While general ERD/ERS procedures analyze this power differential only within specific frequency bands (after bandpassing the EEG signal), ERSP presents a wide band view of the data in the frequency domain [DC-30Hz here] and additionally, statistically quantify the increase/loss in power with respect to baseline. Thus, ERSP are generalizations of ERD/ERS. First, all the epochs for each condition across all subjects were pooled together. Within this pool, epochs corresponding to specific electrodes within each $\mathrm{RO}$ were then extracted and grouped together and the data were resampled. EEG power spectrum $(\mathrm{DC} 30 \mathrm{~Hz})$ was then computed per epoch using a 512 data point $(512 \mathrm{~ms}$ as Fs $=1000 \mathrm{~Hz})$ Fast Fourier Transform (FFT) tapered with a moving Hanning window, $50 \%$ overlap. This window size was chosen to obtain adequate frequency resolution while at the same time corresponding to the window length in the time-voltage analyses (200ms). The first $500 \mathrm{~ms}(-1000-500 \mathrm{~ms})$ was considered as baseline and used to compute significant changes in power ( $p<0.01$, False Detection Rate corrected). The baselined power spectra were averaged across all subjects resulting in grand averaged EEG ERSP for each of the 12 conditions, across all $5 \mathrm{ROI}$. We focused on the effect of the Manipulative hand posture against the No Hand condition within a context, as was done in the time-voltage analyses. To this end, ERSP of the No Hand condition was statistically ( $p<0.001$, uncorrected) compared to the Manipulative posture. Similar to the time-voltage analyses, the hand posture conditions were not directly compared to each other (such as Functional vs. Manipulative). Analyses were primarily restricted to the comparison of the Manipulative posture with respect to 
the No Hand control condition within the correct and spatial contexts. The ROI used in these contrasts were derived from significant findings in the time-voltage statistics and are descried in detail in the subsequent results section.

\section{Results}

\subsection{Time-Voltage Analyses}

For each time window (early, middle and late), a separate 3-way ANOVA was computed with the following factors: Hand (four levels: No hand, Static hand, Functional and Manipulative), Context (three levels: Correct, Incorrect and Spatial) and Region (five levels: Left and Right Frontal, Left Motor, Left and Right Parieto-temporal areas). The 3-way ANOVA computed on the early phase, [100 - 200ms] after picture presentation, revealed a three way interaction of Context $x$ Hand $x$ Region $[F(24,900)=2.26, p<0.05]$, with a main effect of Region $[F(4,900)=$ 551.96, $p<0.05]$ and a two way interaction of Context $x$ Hand $[F(6,900)=8.15, p<0.05]$. The middle phase [200 - 400ms] revealed a significant three way interaction, Context $x$ Hand $x$ Region $[F(24,900)=1.54, p<0.05]$, with a main effect of Region $[F(4,900)=884.88, p<0.05]$ and a significant two way interaction: Context $x$ Hand $[F(6,900)=11.8, p<0.05]$. Analysis on the late phase [400 - 600ms] revealed a significant three way interaction of Context $x$ Hand $x$ Region $[F(24,900)=1.85, p<0.05]$ with a main effects of Region $[F(4,900)=707.92, p<0.05]$ and Hand $[F(3,900)=24.05, p<0.05]$ and the following significant two way interactions: Context $x$ Hand $[F(6,900)=3.86, p<0.05]$, Hand $x$ Region, $[F(12,900)=4.27, p<0.05]$. Given the three way interactions with subsequent main effect of Region and two way interaction of Context $x$ Hand, post-hoc t-tests enabled us to evaluate the contrasts of the various hand conditions (Manipulative, Functional and Static Hand) with respect to the No Hand control condition, within a specific context and over individual ROls across all three time windows. The results of these $\mathrm{ROI}$ analyses are presented in the following sections and Figures 3-5 depict the results. Additionally, 3-way ANOVAs on all conditions confirmed $(p<0.05)$ that i) EEG traces did not differ in the baseline period, ii) EEG waveforms were not divergent at stimulus presentation and iii) convergence of EEG waveform traces of all conditions back to baseline 1.5 seconds post stimulus.

\subsubsection{Left Frontal Area}

\subsubsection{Early phase [100-200ms]}

Since we were primarily concerned with the effect of Hand, we conducted two tailed t-tests within each context, comparing No Hand to other hand conditions. Figure 3A illustrates the results of the left frontal area. When context was Correct, the No Hand posture differed significantly from Static Hand $[\mathrm{t}(30)=6.91, \mathrm{p}<0.01]$, Functional $[\mathrm{t}(30)=3.31, \mathrm{p}<0.01]$ and Manipulative $[\mathrm{t}(30)=3.52, \mathrm{p}<0.01]$ hand postures. When context was Incorrect, only Manipulative differed from No Hand, $[\mathrm{t}(30)=3.97, \mathrm{p}<0.01]$. When context was Spatial, there were no statistical differences among the hand conditions.

\subsubsection{Middle phase [200-400ms]}


In the middle phase, some of the early effects were maintained; two tailed t-tests show that when context was Correct, No Hand differed significantly from Static Hand $[\mathrm{t}(30)=3.11$, $\mathrm{p}<$ $0.01]$ and Manipulative $[\mathrm{t}(30)=2.95, \mathrm{p}<0.01]$ postures. Within the Incorrect context, only the Functional grasp significantly differed from No Hand $[\mathrm{t}(30)=-2.95, \mathrm{p}<0.01]$. Whereas when context was Spatial, there were no statistical differences among the hand conditions.

\subsubsection{Late Phase [400-600ms]:}

Finally, in the late phase, two tailed t-tests show that when context was Correct, there was no statistical difference between hand conditions. When context was Incorrect, No Hand differed significantly from Functional $[\mathrm{t}(30)=-5.53, \mathrm{p}<0.01]$ and Manipulative [t(30) $=-5.68, \mathrm{p}<0.01]$ postures. Uniquely, when context was Spatial, No Hand differed from the Manipulative posture $[\mathrm{t}(30)=-3.82, \mathrm{p}<0.01]$.

\subsubsection{Left Parieto-temporal Area}

\subsubsection{Early Phase [100-200ms]}

Figure 3B illustrates the results of the Left Parieto-Temporal area. When context was Correct, No Hand differed significantly from Static Hand $[\mathrm{t}(30)=4.21, \mathrm{p}<0.01]$, Functional $[\mathrm{t}(30)=4.68$, $\mathrm{p}<0.01$ ] and Manipulative $[\mathrm{t}(30)=5.19, \mathrm{p}<0.01$ ] postures. When context was Incorrect, No Hand differed significantly from Static Hand [t(30] $=3.84, \mathrm{p}<0.01]$, Functional $[\mathrm{t}(30)=2.81, \mathrm{p}<$ $0.01]$ and Manipulative $[\mathrm{t}(30)=3.31, \mathrm{p}<0.01]$ postures. When context was Spatial, No Hand differed significantly from Static Hand $[\mathrm{t}(30)=3.59, \mathrm{p}<0.01]$ and Functional posture $[\mathrm{t}(30)=$ $6.19, p<0.01]$.

\subsubsection{Middle Phase [200-400ms]}

When context was Correct, No Hand differed significantly from Manipulative posture $[\mathrm{t}(30)=$ $3.39, p<0.01]$. When context was Incorrect, No Hand differed significantly from Manipulative $[\mathrm{t}(30)=-2.91, \mathrm{p}<0.01]$ posture. When context was Spatial, No Hand did not differ from any other conditions.

\subsubsection{Late Phase [400-600ms]}

When context was Correct, there we no statistical differences. When context was Incorrect, No Hand differed significantly from Manipulative posture $[\mathrm{t}(30)=-4.99, \mathrm{p}<0.01]$. When context was Spatial, No Hand differed significantly only from the Manipulative posture $[t(30)=-6.8, p<0.01]$.

\subsubsection{Right Frontal Area}

\subsubsection{Early phase [100-200ms]}

Figure 4A illustrates the results of the right frontal area. When context was Correct, the No Hand condition differed significantly from Static Hand posture $[\mathrm{t}(30)=4.99, \mathrm{p}<0.01]$. When context was Incorrect, No Hand differed significantly from Manipulative posture [t(30) $=3.32, p<0.01]$. When context was Spatial, there was no statistical difference among the hand conditions.

\subsubsection{Middle phase [200-400ms]}


There were no statistical differences in the middle phase across all hand postures with respect to No Hand condition.

\subsubsection{Late Phase [400-600ms]:}

Over the right frontal area, two tailed t-tests show that when context was Correct, No Hand differed significantly only from the Manipulative posture [t(30) $=-6.06, p<0.01]$, evidence that the Manipulative posture could drive extended neural processing . When context was Incorrect, No Hand differed significantly from Functional [t(30) $=-5.08, \mathrm{p}<0.01$ ] and Manipulative postures $[\mathrm{t}(30)=-9.14, \mathrm{p}<0.01]$. When context was Spatial, No Hand differed from the Manipulative posture $[\mathrm{t}(30)=-6.16, \mathrm{p}<0.01]$. Figure 4A illustrates these results.

\subsubsection{Right Parieto-temporal Area}

\subsubsection{Early Phase [100-200ms]}

Figure 4B illustrates the results of the Right Parieto-Temporal area. When context was Correct, No Hand differed significantly from Static Hand $[\mathrm{t}(30]=3.61, \mathrm{p}<0.01]$, Functional $[\mathrm{t}(30]=4.65$, $\mathrm{p}<0.01$ ], and Manipulative [t(30) $=5.08, \mathrm{p}<0.01$ ] postures. When context was Incorrect, No Hand differed significantly from Static Hand [t(30] $=5.22, p<0.01]$, Functional $[\mathrm{t}(30)=5.02, \mathrm{p}<$ $0.01]$ and Manipulative $[\mathrm{t}(30)=3.65, \mathrm{p}<0.01]$ postures. When context was Spatial, No Hand differed significantly from Static Hand $[\mathrm{t}(30)=3.06, \mathrm{p}<0.01]$ and Functional posture $[\mathrm{t}(30)=$ 4.52, $p<0.01]$.

\subsubsection{Middle Phase [200-400ms]}

When context was Correct, No Hand differed significantly only from the Manipulative posture $[\mathrm{t}(30)=3.33, \mathrm{p}<0.01]$. When context was Incorrect or Spatial, No Hand did not differ significantly from any other hand condition.

\subsubsection{Late Phase [400-600ms]}

When context was Correct, No Hand differed significantly from Manipulative postures [t(30) = 2.87, $p<0.01$ ]. Together with the Right Frontal, the Right Parieto-temporal area is sensitive to the Manipulative grasp, with extended neural activations. When context was Incorrect, No Hand differed significantly from Manipulative posture [t(30) $=-4.67, \mathrm{p}<0.01]$. When context was Spatial, No Hand differed significantly only from the Manipulative posture $[\mathrm{t}(30)=-6.35, \mathrm{p}<$ 0.01].

\subsubsection{Left Motor}

\subsubsection{Early phase [100-200ms]}

Figure 5 illustrates the results of the left motor area. When context was Correct, No Hand differed significantly from Static Hand $[\mathrm{t}(30)=3.04, \mathrm{p}<0.01]$, Functional $[\mathrm{t}(30)=3.64, \mathrm{p}<0.01]$ and Manipulative postures [t(30) $=4.06, \mathrm{p}<0.01]$. When context was Incorrect, No Hand differed significantly from Static Hand [t(30) $=4.09, \mathrm{p}<0.01]$, Functional $[\mathrm{t}(30)=3.34, \mathrm{p}<0.01]$ and Manipulative postures, [t(30) $=4.05, \mathrm{p}<0.01]$. When context was Spatial, No Hand differed significantly from the Functional posture alone $[\mathrm{t}(30)=3.53, \mathrm{p}<0.01]$. 


\subsubsection{Middle phase [200-400ms]}

When context was Correct, No Hand differed significantly from Manipulative posture $[\mathrm{t}(30)=$ 2.97, $\mathrm{p}<0.01]$. There were no differences when context was Incorrect or Spatial.

\subsubsection{Late phase [400-600ms]}

Focusing on the late phase, when context was Correct, No Hand did not differ significantly from any other hand condition. When context was Incorrect, No Hand differed significantly from Functional $[\mathrm{t}(30)=-4.18, \mathrm{p}<0.01]$ and Manipulative, $[\mathrm{t}(30)=-4.84 \mathrm{p}<0.01]$ postures. When context was Spatial, No Hand differed significantly only from the Manipulative posture $[\mathrm{t}(30)=-$ $6.43, p<0.01$ ] advancing insight into the neural processing of the Manipulative grasp and the Spatial context.

\subsection{Time Frequency Analysis}

The time varying ERSP of the EEG signal [DC 30Hz] was computed to investigate outcomes in frequency power. Only ROls with significant differences in the time-voltage analyses that were relevant to our hypotheses were analyzed. Using ERSP plots, we were able to identify distinct changes in power within unique bands. Statistical differences in the ERSP for the Correct and Spatial contexts when Manipulative was compared to the No Hand condition are shown in Figures 6 and 7. Individual ERSPs are shown as supplementary Figures (1-7).

3.2.1 Correct context: Over the right frontal area, when context was Correct, the Manipulative posture had significantly more positive differences in power ( $p<0.001$, uncorrected) when compared to the No Hand condition, specific to theta and low alpha frequency bands [4-10Hz]. This modulation emerges early and sustains throughout all time-windows of interest as can be seen in Figure 6A. Over the right parietal area, Manipulative posture exhibited both positive and negative differentials. In the beta band $[15-20 \mathrm{~Hz}]$, No Hand condition had more negative ERSP than Manipulative; therefore (Manipulative - No Hand) resulted in a positive differential (see figure caption for detailed explanation). In alpha band [8-10Hz], Manipulative exhibited a larger negative ERSP (ERD) than No Hand, giving rise to a negative differential (Supplementary Figure 2). These modulations were present only in the late window ( $p<0.001,[400-600 \mathrm{~ms}])$ and the statistical differences are shown in Figure 6B.

3.2.2. Spatial context: The Manipulative posture alone engages bilateral parietofrontal networks and the left motor area, with significantly greater beta band ERD than No Hand [12-16 Hz, 20$25 \mathrm{~Hz}$ ], resulting in an overall negative differential (Supplementary Figures 3-7). These power differences are present only in the late phase $(p<0.001$, [400-600ms], uncorrected) and are shown in Figure 7.

\section{Discussion:}

Our earlier behavioral study (Borghi, et al., 2012) revealed an interaction between hand posture and context when judging tool-object images. Within the Correct context, subjects exhibited slowest judgments when hand posture was Manipulative. Within the Spatial context, the Manipulative and Functional hand postures elicited the slowest judgment times when compared to No Hand. As well, it was confirmed that object-recognition in the experiment was not impaired by the type of hand posture; this ruled out effect of interference or clutter in the visual stimuli. 
Participants' response times were not influenced by any artefactual properties of the various hand postures. Taken with the present results, tool-object relationship and hand posture are together important in perceptual judgments of action. Specifically in the present study, the neural mechanism underlying contextual tool-object perception can be affected simply by varying the hand posture. We analyze the effect of hand posture within a context, to answer our main hypotheses. The discussion is based on the post-hoc tests to examine spatiotemporal neural processing of each hand condition with respect to the No Hand condition. Within the Correct context in the early phase (100-200ms), Functional and Manipulative differed from No Hand over left parietofrontal, left motor and right parietal areas, while the Static Hand differed bilaterally over parietofrontal and left motor areas. The Functional was not significant with respect to the No Hand at time scales $>200 \mathrm{~ms}$. In the middle phase ( 400ms), the Manipulative condition continued to elicit differences over same ROI as in the early phase, while the Static Hand was restricted to left frontal area. Finally, in the late phase, the only significant result was the right parietofrontal circuit for the Manipulative Posture at time scales relevant to our prior behavioral result ( $600 \mathrm{~ms})$ and thereby answering our first and second hypotheses, where we had posited that the Manipulative posture alone would have increased and prolonged left parietofrontal activity with respect to the No Hand. The EEG waveform traces for this condition were thus analyzed in the frequency domain to identify significant ERSP, with respect to the No Hand. Similarly, when tool object context was Spatial, the Manipulative posture alone was characterized by extended ( 600ms) bilateral parietofrontal and left motor differences in timevoltage and time-frequency domains, while Functional and Static Hand elicited only early bilateral parietal, left motor and bilateral parietal activity respectively. The presence of distinct parietofrontal activations in the Spatial context addresses our third hypothesis where we had posited that these neural effects would be restricted to the Correct context alone where action affordance was highest. These results offer a neural explanation of the behavioral outcomes seen in our prior work, and introduce potential neural mechanisms outside of the left parietofrontal network commonly engaged in deciphering tools and tool-object based action (L. Chao \& Martin, 2000; S. Johnson-Frey, et al., 2005; S. H. Johnson-Frey, 2004; Kroliczak \& Frey, 2009; Martin, 2007). Direct comparisons between all conditions (Manipulative and Functional for example), has not been carried out as it is not our primary hypotheses and would require an exceedingly large number of post-hoc tests. However, we contrast the latencies of the neural differences of the various hand conditions with respect to the No Hand in our discussion. Here, we discuss the results in light of our original hypotheses.

\subsection{Do manipulative postures evoke increased left parietofrontal activity for image evaluation?}

We hypothesized that observing a Manipulative tool grasp when tool and object functionally matched would significantly engage left parietofrontal activity when compared to the No Hand condition, indicative of increasing processing of tool-use action network. Data did confirm our hypothesis of this increased early activity; in fact, the Manipulative posture was significantly active over bilateral parietal, left motor and left frontal areas [100-400ms]. However, only right parietofrontal activity persisted later, in time windows corresponding to our behavioral study [400-600ms]. Notably, the right frontal area involvement was an emergent feature only in the late phase. This right network also involved theta synchronization over the right frontal area. Therefore, we propose there are two networks at work: an early left parietofrontal-motor network to process the familiar tool-object association and a right parietofrontal network to decode the unusual Manipulative posture, with common right parietal activity throughout. We will detail activations related to established literature while discussing the second hypothesis. 
4.2 Do manipulative postures generate prolonged left parietofrontal activation for image evaluation?

A highlight of our previous work was seen for Manipulative postures in the Correct tool object context, which required the longest judgment times (Borghi, et al., 2012). Based on this behavioral result, we had hypothesized that this image class would elicit prolonged left parietofrontal activations as subjects performed similar tool-object judgments in the present EEG study. In contrast, the Manipulative posture exhibited significant late activations over right parietofrontal areas. Additionally, in the time-frequency domain, this extended neural processing primarily corresponded to modulations in the theta $[4-8 \mathrm{~Hz}]$ and low alpha $[8-10 \mathrm{~Hz}]$ bands over the right frontal areas and modulations in low alpha and beta bands [15-20Hz] over right parietal areas. Thus, the Manipulative posture seems to engage two neural systems at different times relative to the stimulus onset: an early left parietofrontal, left motor and right parietal activation that transitions to an extended late right parietofrontal activation. We propose that the first network identifies functional tool object associations that are possible (Mizelle \& Wheaton, 2010c), before activations transition to the extended late right parietofrontal network that could underlie different cognitive motor processes, as proposed below.

\subsubsection{Potential Role of Response Inhibition}

A core idea of behavioral processes is the mechanisms of 'response selection' and 'response inhibition' (Ridderinkhof, Ullsperger, Crone, \& Nieuwenhuis, 2004; Ridderinkhof, Van Den Wildenberg, Segalowitz, \& Carter, 2004). It is equally important to understand when to not execute a task. Inhibitory control and error detection are therefore a very important aspect of human cognitive control (Rubia, Smith, Brammer, \& Taylor, 2003). Recently, Goghari et al identified that the right inferior frontal gyrus was found to be heavily involved when performing a task that required response inhibition (Goghari \& MacDonald III, 2009). As well, using a Go/NoGo paradigm, Rubia et al isolated the right inferior prefrontal cortex to mediate motor response inhibition (Rubia, et al., 2003). From a cognitive motor control standpoint, the right prefrontal areas appear to be responsible for higher level motor response inhibition. Right frontal activation has also been seen in EEG studies of tool use pantomime in the Go/No-Go paradigm (L. Wheaton, Fridman, Bohlhalter, Vorbach, \& Hallett, 2009). In the present study, significant activity was found late over the right frontal area, when subjects judged tool object images placed in a Correct context with a Manipulative hand posture. When a tool-object pair forms a functional match, it is uncommon to grasp the non-functional end of the tool. For example, it is does not make subjective sense to grasp the sharp end of the knife when paired with a slab of butter. This act would be perceived as unusual, as well as affording the least object-driven action. In this case, right frontal activity may correspond to inhibiting a final cognitive decision of the tool-object pair thus prolonging the decision making process. However, inhibition may not be the driving mechanism. In the behavioral work (Borghi, et al., 2012) it was shown that the Manipulative posture delay was present when subjects pressed the response button with their hand, but was absent when subjects were required to respond via foot switches. If inhibition were at the decision-making level, it should not be sensitive to how the responses were made (i.e., not limb specific). Thus, another mechanism could be involved as described below.

\subsubsection{Mirror Neuron System}


The existence of specific parietofrontal networks that are active when performing and observing similar hand-object actions have been well documented (Rizzolatti \& Craighero, 2004; Rizzolatti \& Sinigaglia, 2010). This network has been called the mirror neuron network and its existence had been initially discovered in the primate brain, with subsequent human analogues. Activity of this network overlaps with areas responsible for tools and tool based action (Lewis, 2006). In our study, we found differential recruitment of parietofrontal circuits that seem to be proportional to the action afforded in the image e.g., extended right activity in response to the Manipulative posture when compared to No Hand. This network is distinct from the canonical neuron system that underlies processing of prehensile motions on observations of graspable objects (Raos, Umiltá, Murata, Fogassi, \& Gallese, 2006). The canonical system might be particular to the No Hand condition while the Manipulative Hand condition would additionally elicit the mirror neuron system.

While many studies have demonstrated that the mirror neuron system is largely left hemispheric, several studies have suggested right hemispheric involvement (Aziz-Zadeh, Koski, Zaidel, Mazziotta, \& lacoboni, 2006; M. lacoboni \& Dapretto, 2006). Of interest is the fMRI experiment of lacoboni et al. (2005) who studied the mirror neuron system employing a paradigm that shares common premises with the present EEG study. Their goal was to understand the role of the mirror neuron system in complex action understanding, specifically in deciphering intent of an observed action. The authors demonstrated that the posterior right inferior frontal gyrus was significantly active in deciphering goal or intent when subjects viewed the same grasping action embedded in two different contexts (pick up to drink vs. pick up to clean) (M lacoboni et al., 2005). Other studies have also found similar right hemispheric activation attributed to the mirror neuron system (S. H. Johnson-Frey, 2004; Villarreal et al., $\underline{2008}$ ). Therefore, our results here might be indicative of a late right hemispheric mirror neuron response for deciphering intent of the unusual Manipulative grasp when context is correct. Since the common Functional grasp is already familiar, it may trigger early activity primarily over left parietofrontal and motor networks, areas which store pre-existing templates for the observed action (Bohlhalter et al., 2008; Mizelle \& Wheaton, 2010c; L. Wheaton, et al., 2009). Whereas the Manipulative posture, being an uncommon grasp when tool-use context is Correct, can additionally engage right parietofrontal areas to decipher the intent or goal of the action. It should be noted that the right frontal area differs from No Hand only in the late time scales, while the right parietal area differs throughout, early with the left network and then late at right frontal areas. While this temporal difference might suggest that the right parietal activation might precede right frontal activation, ERSP reveal a duality wherein right frontal activity precedes right parietal activity at specific frequency bins with respect to No Hand. These frequency characteristics are detailed in the next section. Our results here directly question previous findings showing that bilateral inferior frontal regions do not distinguish functionally grasping vs. non-functionally grasping (kinetically similar to our Manipulative grasp) a tool ( $\underline{\mathrm{S}} \mathrm{H}$. JohnsonFrey et al., 2003). In that particular study, the tool was always presented stand-alone, and either functionally or non-functionally grasped. Here, we show that when the tool is paired with a functionally appropriate object, then non functional grasps do elicit frontal differences with high temporal resolution, and strengthen our argument that context and hand posture together modulate tool-object perception. As well, engagement of this mirror neuron network suggests that participants decipher the Manipulative grasp in relation to the tool-object pairing without any apparent need to perform a mental rotation of this unusual hand posture. We are performing further experiments on the same set of stimuli where it may be possible to infer if the grasp posture draws significant visual attention to address any issue of mental rotation of hand posture. 


\subsubsection{Role of Low Frequency Power Modulations}

Time frequency analyses revealed additional outcomes of neural processing when context was Correct and hand posture was Manipulative (Figure 6), primarily in theta and low alpha bands over the right frontal area. This unique difference starts at image onset and persists for $500 \mathrm{~ms}$. Over the right parietal areas, the Manipulative posture alone had significant positive differentials in beta bands and negative differentials in low alpha bands. However, these modulations were present only later in time ( 400-600ms post image), presenting a duality with the time-voltage results wherein right parietal differences preceded right frontal. This temporal lag might be indicative of the role of the right prefrontal cortex as a driver of right parietal activity at specific frequency bins, especially when updating knowledge of a cognitive task (Brass, Ullsperger, Knoesche, Cramon, \& Phillips, 2005). In addition, theta synchronization has recently been implicated in having a role in the mirror neuron system via Hebbian learning mechanisms (Del Giudice, Manera, \& Keysers, 2009) and may be involved in motor imagery and decoding sensori-motor aspects of the stimuli (Caplan et al., 2003) or as an indicator of executive control in cognitive interference (Nigbur, Ivanova, \& Stürmer, 2011). While concrete links to these concepts and our work is premature, it may demonstrate a mechanism whereby specific frequency oscillations serve to bind action perception with action memories. This particular question is being addressed in ongoing studies.

\subsection{Does the presence of a hand influence neural activation in each context?}

\subsubsection{Correct context}

In the Correct context, the Functional and Manipulative postures had significantly different early activations from No Hand over the left parietofrontal, left motor and right parietal areas, with Static Hand exhibiting early bilateral parietofrontal and left motor differences. Therefore, the mere presence of a hand (in any posture) when tool object context was Correct triggered an increased early neural activation over parietofrontal areas, with Functional and Manipulative postures having increased activity over predominantly left areas. Since subjects were instructed to judge the correctness of the images, we speculate that this activity underlies deciphering toolobject action (Lewis, 2006). The fact that the No Hand condition was significantly different from all other Hand conditions speaks directly to our previous behavioral results wherein the No Hand condition in the Correct tool object context produced the fastest reaction times.

On transitioning into the middle phase [200-400ms post image], the Manipulative posture had continued activations similar to the early phase (left parietofrontal, left motor and right parietal), indicating ongoing decision on tool-object action. On the other hand, the Functional posture did not differ from the No Hand in this or the late phase, possibly indicating early completion of simulation of action affordances, due to the appropriateness of the Correct context and the Functional grasp. Finally, the Static Hand condition elicited significant differences only over left frontal area. This might be a consequence of the open-ended nature of the Static Hand condition, wherein the hand is positioned in a neutral posture relative to the tool. This would strongly engage frontal areas (Hamilton \& Grafton, 2008; Heyes, 2001; Pobric \& de C. Hamilton, $\underline{2006}$ ) if subjects visualize and predict end points of a potential tool grasp. As detailed in the previous section, only the Manipulative posture was significant over right parietofrontal network later in time. The time scales of these activations speak to our prior behavioral results wherein this posture alone elicited the longest judgment times. This right network therefore underlies processing of unfamiliar tool-object action. As well, these results address our first hypothesis wherein we had posited that the Manipulative posture alone would have stronger and extended 
left activity relative to the No Hand condition. We find this to be true; Manipulative alone has left parietofrontal, left motor and right parietal activity until 400ms when compared to No Hand. This was not the case with the other hand contrasts. However, in the late phase, this primarily left activity transitioned into an extended right parietofrontal activity, nullifying our second hypothesis where we had posited that the left network would exhibit extended activity.

\subsubsection{Spatial context}

Our final hypothesis stated that any effects of hand posture would be restricted to the Correct context, where action affordance was highest due to the tool-object relationship. However, this was not the case in the Spatial context, wherein the differences directly speak to our prior behavioral results. Notably, the Manipulative posture alone elicited significant differences from the No Hand condition over bilateral parietofrontal and left motor areas. Moreover, these differences surfaced only in the late phase (400-600ms post image), while being similar until $400 \mathrm{~ms}$. We theorize this delay to be attributed to the ambiguous nature of the tool-object relationship where action affordances are not immediately apparent, with or without the Manipulative posture that ostensibly does not affect early processing of affordances. The late bilateral parietofrontal and left motor differences in both time-voltage and time-frequency domains underlie two networks at play in evaluating the image: a right parietofrontal network to comprehend the unfamiliar hand action (presumably right mirror neuron networks) and a left parietofrontal, motor network to decode possible action in the spatial context. In the frequency domain, increased ERD was present for the Manipulative posture vs. No Hand over the aforementioned areas [ $p<0.001$, uncorrected, 400-600ms post image] dominant within two frequency bins $[12-17 \mathrm{~Hz}$ and $20-25 \mathrm{~Hz}]$. The beta band ERSP effects emerge at the same time as the time-voltage effects, indicating an absence of the duality observed in the Correct context for the same Manipulative posture. This is in line with previous reports showing parietofrontal voltage and beta band activity related to understanding tool-use and tool-use simulations (Mizelle, et al., 2011). Therefore, similar to the Correct context, we propose that the Manipulative posture in the Spatial context also engages two neural systems, though with differing time scales and spectral properties arising from the ambiguous tool-object relationship, that directly addresses behavioral data and our final hypothesis. Unlike Correct or Incorrect, the Spatial context is distinctive in presenting action affordances that are not immediately obvious. Related to the tool action itself, extended bilateral parietofrontal activity serves to continually evaluate the content in a unique way beyond when the context is Correct. Use of bilateral parietofrontal networks continually in the late phase would permit for ongoing evaluation of the intent of the action scene (M lacoboni, et al., 2005), and perhaps ongoing activation related to the content of the scene itself (L. L. Chao, et al., 2002; Weisberg, et al., 2007), to determine if action is possible between the tools and objects as discrete entities. The Spatial context is also unique in presenting a functional relationship with regard to being used in conjunction bimanually or in sequence. For example, in our exemplar stimuli, the coffeepot and milk carton can be used to make coffee, though one does not normally use them directly on each other. Our results here could possibly explain an aspect of the recent study by DeStefani et al. (De Stefani, Innocenti, Bernardi, Campione, \& Gentilucci, 2012) who did not find an interference effect when participants picked up a bottle in the presence of a distractor such as an apple or sphere. It could very well be that the apple bears little functional relationship with the bottle, even indirectly. A possible replacement for the apple would have been a spatially related object. As well, varying the grasp might have altered the interference effect. 
The other hand conditions, Static Hand and Functional, differed from No Hand only in the early phase over bilateral parietal areas, with Functional being different over left motor as well. These early parietal differences can be attributed to the action afforded by either Functional or Static Hand posture given the possibility of engaging the tool on the object. Even though the Spatial context of tool-object relationship is ambiguous by nature, grasping the tool functionally still affords action (Buxbaum \& Kalénine, 2010). Such action affordance may be similar to proposed models of atypical tool-use, which would also necessitate engagement of tool action representations in the parietal and left motor areas (Mizelle \& Wheaton, 2010b). In addition, the Functional posture exhibited weak differences over left parietofrontal areas in the late phase $(p<0.05)$. Though this effect did not reach significance, it can possibly explain our prior behavioral result where the Functional grasp did elicit longer response times when compared to the No Hand when context was Spatial. On the other hand, The Static Hand condition, though open ended, engages neural systems similar to the Functional posture. This is in contrast to the Correct context, where the Static Hand condition differed from No Hand over frontal areas, notably left frontal. Thus the effect of this hand posture is significantly altered by the tool-object context. These results address our third hypothesis and show that though action affordances change based on context, the combination of hand posture and context together modulate neural activity underlying increased processing of tool-object action.

\section{$\underline{4.3 .3 \text { Incorrect context }}$}

We have originally proposed that incorrect tool-object relationships would insufficiently activate parietofrontal activations, as evaluation of the tools and objects would facilitate ventral stream activation which would not facilitate action encoding of incongruent objects (Mizelle, et al., 2011; Mizelle \& Wheaton, 2010a) an idea that has been proposed by other investigators (Valyear \& Culham, 2010). In addition, this context failed to produce any significant results in our prior behavioral study. Thus, in the present paradigm, the grasp posture effects seen may be weakened if the tool-object relationship is not sufficient to drive action simulation mechanisms. However, parietofrontal activity does emerge in this context for the various hand conditions. The Manipulative posture differs from No Hand over bilateral parietal and left motor areas early, while Static Hand and Functional differ from No Hand only over bilateral parietal and left motor areas in the same period $(100-200 \mathrm{~ms})$. The middle phase does not produce any patterns of interest. The late phase $(400-600 \mathrm{~ms})$ is characterized by bilateral parietofrontal and left motor differences for the Manipulative posture, and only left motor and bilateral frontal differences for the Functional posture. Thus even in the Incorrect context, the varying hand posture do elicit significant patterns of parietofrontal activity. However, our main hypotheses concerned our prior behavioral findings, wherein this context failed to produce any reliable results. Nevertheless, these results address our third hypothesis: the combination of hand grasp and context offsets the lower action affordances due to tool-object relationship alone and elicits parietofrontal activity. Further work on processing of incorrect tool-object relationships is underway to better evaluate and understand this finding.

\section{$\underline{\text { 5. Conclusions }}$}

Our goal was to understand the neural bases of our initial behavioral study (Borghi, et al., 2012) of tool-use perception considering grasp posture and context. We hypothesized that observing a Manipulative tool grasp when tool and object functionally matched would engage continuous left parietofrontal activity, indicative of increasing processing of tool-use action, and hence, longer 
judgment times, while Static Hand and Functional would elicit only early left differences when compared to No Hand. While we did see some predominantly left parietofrontal activity early for all three comparisons, it turned out that only the Manipulative posture had stronger left activity $(\sim 400 \mathrm{~ms})$ before transition to extended neural activity over a right parietofrontal network with time scales ( 600ms) fitting the behavioral results, with a late appearing right frontal. The latter exhibited a duality in the time-frequency domain with early theta synchronization while there was late beta desynchronization over the right parietal area. We propose that there are two parietofrontal networks underlying perception: a predominantly early left parietofrontal network to process the familiar tool-object association and a later right parietofrontal network to decode the unusual Manipulative posture. As well, we also found that the Manipulative posture in the Spatial context engaged bilateral parietofrontal areas in later time windows fitting behavioral data. We posit that the late right parietofrontal network is engaged to understand action intent of the Manipulative posture, and the left parietofrontal activity is used to comprehend the possible interactions of tools and objects themselves in the scene. These networks exhibit time locked local desynchronization in beta bands to meet the increased tool-use simulation demands in understanding the Manipulative grasp when context is Spatial.

While this work provides unique context and grasp variable neural responses to action perception, further studies are needed to understand why temporal delays in behavior and neural activity result. In future work, we aim to further explore how the visual system integrates scene elements, and how context and grasp variability can affect perception in a precise temporal scale.

\section{$\underline{\text { References }}$}

Aziz-Zadeh, L., Koski, L., Zaidel, E., Mazziotta, J., \& lacoboni, M. (2006). Lateralization of the human mirror neuron system. The Journal of Neuroscience, 26(11), 2964.

Beauchamp, M. S., \& Martin, A. (2007). Grounding object concepts in perception and action: evidence from fMRI studies of tools. Cortex, 43(3), 461-468.

Bohlhalter, S., Hattori, N., Wheaton, L., Fridman, E., Shamim, E., Garraux, G., et al. (2008). Gesture subtype-dependent left lateralization of praxis planning: an event-related fMRI study. Cerebral Cortex.

Borghi, A. M., Flumini, A., Natraj, N., \& Wheaton, L. A. (2012). One hand, two objects: Emergence of affordance in contexts. Brain and Cognition, 80(1), 64-73. 
Brass, M., Ullsperger, M., Knoesche, T. R., Cramon, D. Y., \& Phillips, N. A. (2005). Who comes first? The role of the prefrontal and parietal cortex in cognitive control. Journal of Cognitive Neuroscience, 17(9), 1367-1375.

Bub, D. N., Masson, M. E. J., \& Cree, G. S. (2008). Evocation of functional and volumetric gestural knowledge by objects and words. Cognition, 106(1), 27-58.

Buxbaum, L. J., \& Kalénine, S. (2010). Action knowledge, visuomotor activation, and embodiment in the two action systems. Annals of the New York Academy of Sciences, 1191(1), 201-218.

Caplan, J. B., Madsen, J. R., Schulze-Bonhage, A., Aschenbrenner-Scheibe, R., Newman, E. L., \& Kahana, M. J. (2003). Human oscillations related to sensorimotor integration and spatial learning. The Journal of Neuroscience, 23(11), 4726.

Chao, L., \& Martin, A. (2000). Representation of manipulable man-made objects in the dorsal stream. Neuroimage, 12(4), 478-484.

Chao, L. L., Weisberg, J., \& Martin, A. (2002). Experience-dependent modulation of categoryrelated cortical activity. Cerebral Cortex, 12(5), 545-551.

Costantini, M., Ambrosini, E., Scorolli, C., \& Borghi, A. M. (2011). When objects are close to me: Affordances in the peripersonal space. Psychonomic bulletin \& review, 18(2), 302-308.

De Stefani, E., Innocenti, A., Bernardi, N. F., Campione, G. C., \& Gentilucci, M. (2012). The bottle and the glass say to me:"Pour!". Experimental Brain Research, 1-11.

Del Giudice, M., Manera, V., \& Keysers, C. (2009). Programmed to learn? The ontogeny of mirror neurons. Developmental Science, 12(2), 350-363.

Delorme, A., \& Makeig, S. (2004). EEGLAB: an open source toolbox for analysis of single-trial EEG dynamics including independent component analysis. Journal of neuroscience methods, 134(1), 9-21.

Efron, B. (1981). Nonparametric estimates of standard error: The jackknife, the bootstrap and other methods. Biometrika, 68(3), 589.

Falkenstein, M., Hoormann, J., \& Hohnsbein, J. (2001). Changes of error-related ERPs with age. Experimental Brain Research, 138(2), 258-262.

Goghari, V. M., \& MacDonald III, A. W. (2009). The neural basis of cognitive control: response selection and inhibition. Brain and Cognition, 71(2), 72-83.

Gómez-Herrero, G. (2007). Automatic Artifact Removal (AAR) toolbox v1. 3 (Release 09.12. 2007) for MATLAB.

Grezes, J., \& Decety, J. (2002). Does visual perception of object afford action? Evidence from a neuroimaging study. Neuropsychologia, 40(2), 212-222. 
Hamilton, A. F. C., \& Grafton, S. T. (2008). Action outcomes are represented in human inferior frontoparietal cortex. Cerebral Cortex, 18(5), 1160-1168.

Hayes, M. (2009). Statistical digital signal processing and modeling: Wiley-India.

He, P., Wilson, G., \& Russell, C. (2004). Removal of ocular artifacts from electro-encephalogram by adaptive filtering. Medical and Biological Engineering and Computing, 42(3), 407-412.

Heyes, C. (2001). Causes and consequences of imitation. Trends in Cognitive Sciences, 5(6), 253261.

lacoboni, M., \& Dapretto, M. (2006). The mirror neuron system and the consequences of its dysfunction. Nature Reviews Neuroscience, 7(12), 942-951.

lacoboni, M., Molnar-Szakacs, I., Gallese, V., Buccino, G., Mazziotta, J., \& Rizzolatti, G. (2005). Grasping the intentions of others with one's own mirror neuron system. PLoS Biol, 3(3), e79.

Jacquet, P. O., Chambon, V., Borghi, A. M., \& Tessari, A. (2012). Object Affordances Tune Observers' Prior Expectations about Tool-Use Behaviors. PloS one, 7(6), e39629.

Jasper, H. H. (1958). The ten-twenty electrode system of the International Federation. Electroencephalography and Clinical Neurophysiology, 10(2), 371-375.

Jeannerod, M., Arbib, M. A., Rizzolatti, G., \& Sakata, H. (1995). Grasping objects: the cortical mechanisms of visuomotor transformation. Trends in Neurosciences, 18(7), 314-320.

Johnson-Frey, S., Newman-Norlund, R., \& Grafton, S. (2005). A distributed left hemisphere network active during planning of everyday tool use skills. Cerebral Cortex, 15(6), 681.

Johnson-Frey, S. H. (2004). The neural bases of complex tool use in humans. Trends in Cognitive Sciences, 8(2), 71-78.

Johnson-Frey, S. H., Maloof, F. R., Newman-Norlund, R., Farrer, C., Inati, S., \& Grafton, S. T. (2003). Actions or hand-object interactions? Human inferior frontal cortex and action observation. Neuron, 39(6), 1053-1058.

Kroliczak, G., \& Frey, S. (2009). A common network in the left cerebral hemisphere represents planning of tool use pantomimes and familiar intransitive gestures at the handindependent level. Cerebral Cortex.

Lewis, J. W. (2006). Cortical networks related to human use of tools. The Neuroscientist, 12(3), 211.

Liavas, A. P., \& Regalia, P. A. (1999). On the numerical stability and accuracy of the conventional recursive least squares algorithm. Signal Processing, IEEE Transactions on, 47(1), 88-96. 
Mahon, B., Milleville, S., Negri, G., Rumiati, R., Caramazza, A., \& Martin, A. (2007). Actionrelated properties shape object representations in the ventral stream. Neuron, 55(3), 507-520.

Makeig, S. (1993). Auditory event-related dynamics of the EEG spectrum and effects of exposure to tones. Electroencephalography and Clinical Neurophysiology, 86(4), 283293.

Makeig, S., Debener, S., Onton, J., \& Delorme, A. (2004). Mining event-related brain dynamics. Trends in Cognitive Sciences, 8(5), 204-210.

Martin, A. (2007). The representation of object concepts in the brain. Annual Review of Psychology, 58, 25-45.

Mizelle, J., Tang, T., Pirouz, N., \& Wheaton, L. A. (2011). Forming Tool Use Representations: A Neurophysiological Investigation into Tool Exposure. Journal of Cognitive Neuroscience(Early Access), 1-15.

Mizelle, J., \& Wheaton, L. A. (2010a). Neural activation for conceptual identification of correct versus incorrect tool-object pairs. Brain Research.

Mizelle, J., \& Wheaton, L. A. (2010b). The Neuroscience of Storing and Molding Tool Action Concepts: How "Plastic" is Grounded Cognition? Frontiers in Psychology, 1.

Mizelle, J., \& Wheaton, L. A. (2010c). Why is that Hammer in My Coffee? A Multimodal Imaging Investigation of Contextually Based Tool Understanding. Frontiers in Human Neuroscience, 4.

Nieuwenhuis, S., Forstmann, B. U., \& Wagenmakers, E. J. (2011). Erroneous analyses of interactions in neuroscience: a problem of significance. Nature neuroscience, 14(9), 1105-1107.

Nigbur, R., Ivanova, G., \& Stürmer, B. (2011). Theta power as a marker for cognitive interference. Clinical Neurophysiology.

Pellicano, A., lani, C., Borghi, A. M., Rubichi, S., \& Nicoletti, R. (2010). Simon-like and functional affordance effects with tools: The effects of object perceptual discrimination and object action state. The Quarterly Journal of Experimental Psychology, 63(11), 2190-2201.

Pfurtscheller, G., \& Lopes da Silva, F. (1999). Event-related EEG/MEG synchronization and desynchronization: basic principles. Clinical Neurophysiology, 110(11), 1842-1857.

Pobric, G., \& de C. Hamilton, A. (2006). Action understanding requires the left inferior frontal cortex. Current Biology, 16(5), 524-529.

Raos, V., Umiltá, M. A., Murata, A., Fogassi, L., \& Gallese, V. (2006). Functional properties of grasping-related neurons in the ventral premotor area F5 of the macaque monkey. Journal of neurophysiology, 95(2), 709-729. 
Ridderinkhof, K. R., Ullsperger, M., Crone, E. A., \& Nieuwenhuis, S. (2004). The role of the medial frontal cortex in cognitive control. Science, 306(5695), 443.

Ridderinkhof, K. R., Van Den Wildenberg, W. P. M., Segalowitz, S. J., \& Carter, C. S. (2004). Neurocognitive mechanisms of cognitive control: the role of prefrontal cortex in action selection, response inhibition, performance monitoring, and reward-based learning. Brain and Cognition, 56(2), 129-140.

Rizzolatti, G., \& Craighero, L. (2004). The mirror-neuron system. Annu. Rev. Neurosci., 27, 169192.

Rizzolatti, G., \& Sinigaglia, C. (2010). The functional role of the parieto-frontal mirror circuit: interpretations and misinterpretations. Nat Rev Neurosci, 11(4), 264-274.

Rubia, K., Smith, A. B., Brammer, M. J., \& Taylor, E. (2003). Right inferior prefrontal cortex mediates response inhibition while mesial prefrontal cortex is responsible for error detection. Neuroimage, 20(1), 351-358.

Steffensen, S. C., Ohran, A. J., Shipp, D. N., Hales, K., Stobbs, S. H., \& Fleming, D. E. (2008). Gender-selective effects of the P300 and N400 components of the visual evoked potential. Vision research, 48(7), 917-925.

Valyear, K. F., \& Culham, J. C. (2010). Observing learned object-specific functional grasps preferentially activates the ventral stream. Journal of Cognitive Neuroscience, 22(5), 970-984.

Van Elk, M., Van Schie, H. T., Van Den Heuvel, R., \& Bekkering, H. (2010). Semantics in the motor system: motor-cortical beta oscillations reflect semantic knowledge of endpostures for object use. Frontiers in Human Neuroscience, 4.

Villarreal, M., Fridman, E. A., Amengual, A., Falasco, G., Gerscovich, E. R., Ulloa, E. R., et al. (2008). The neural substrate of gesture recognition. Neuropsychologia, 46(9), 23712382.

Weisberg, J., van Turennout, M., \& Martin, A. (2007). A neural system for learning about object function. Cereb Cortex, 17(3), 513-521.

Wheaton, L., Fridman, E., Bohlhalter, S., Vorbach, S., \& Hallett, M. (2009). Left parietal activation related to planning, executing and suppressing praxis hand movements. Clinical Neurophysiology, 120(5), 980-986.

Wheaton, L. A., Nolte, G., Bohlhalter, S., Fridman, E., \& Hallett, M. (2005). Synchronization of parietal and premotor areas during preparation and execution of praxis hand movements. Clinical neurophysiology: official journal of the International Federation of Clinical Neurophysiology, 116(6), 1382. 


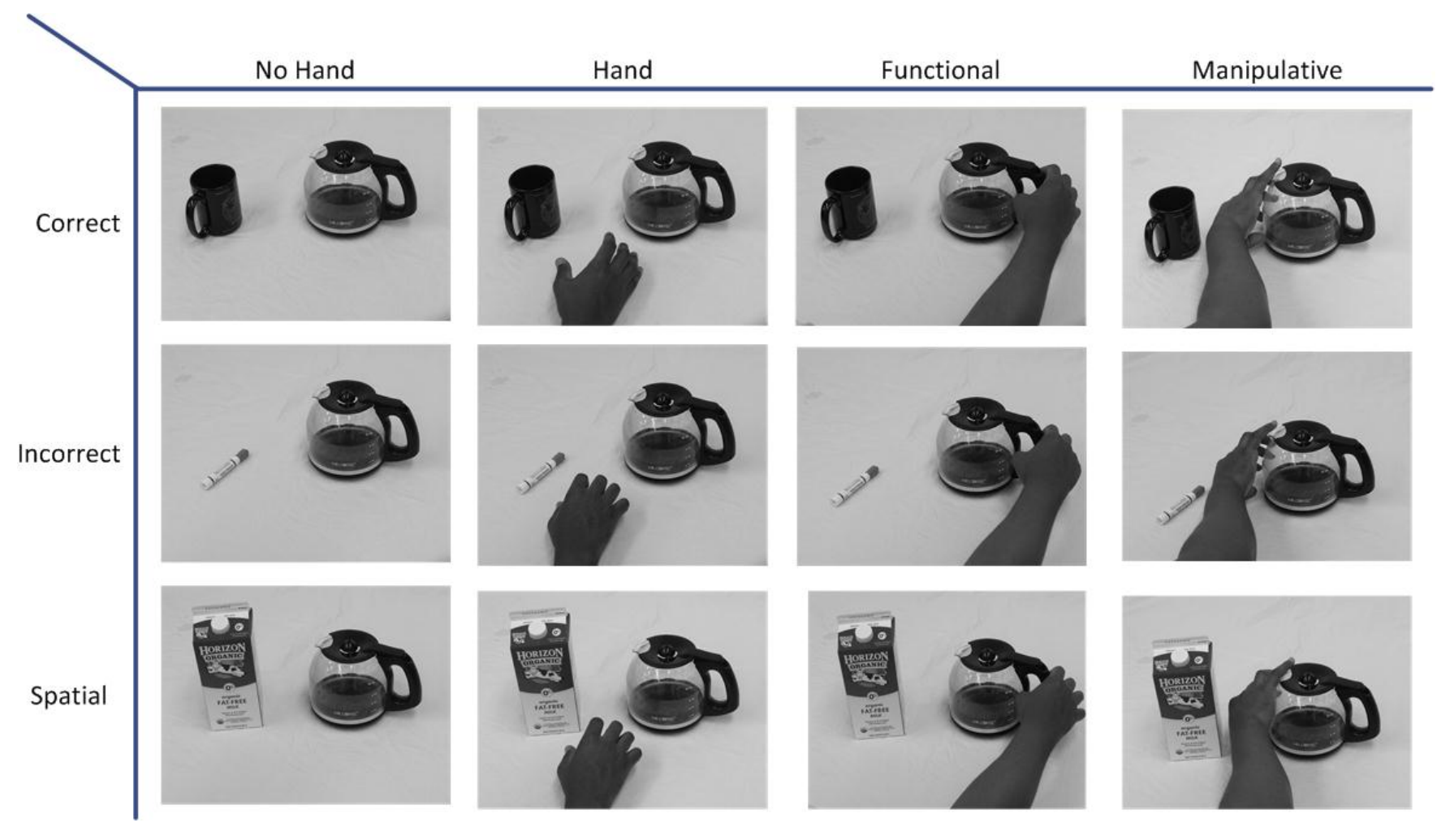



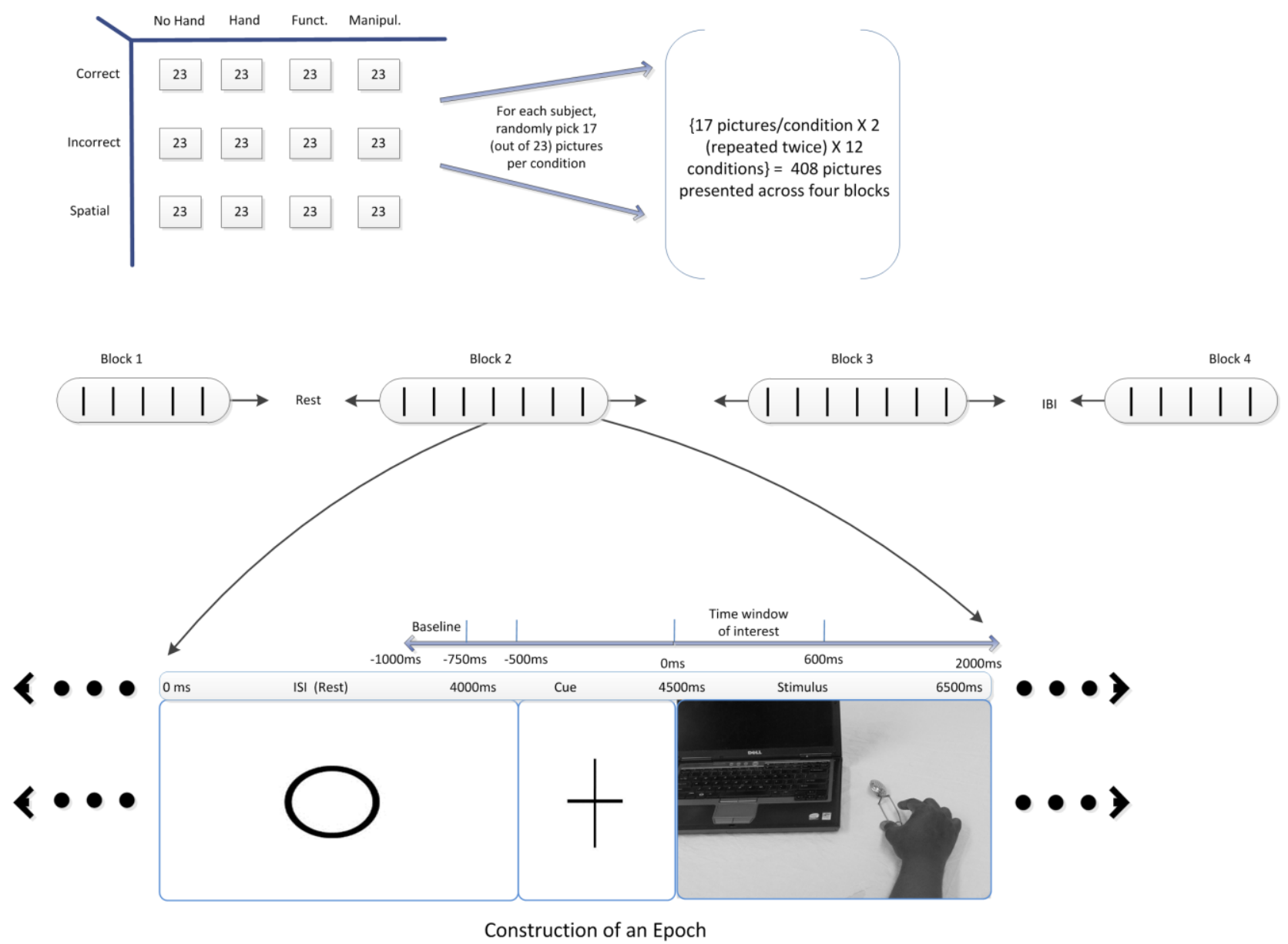
A
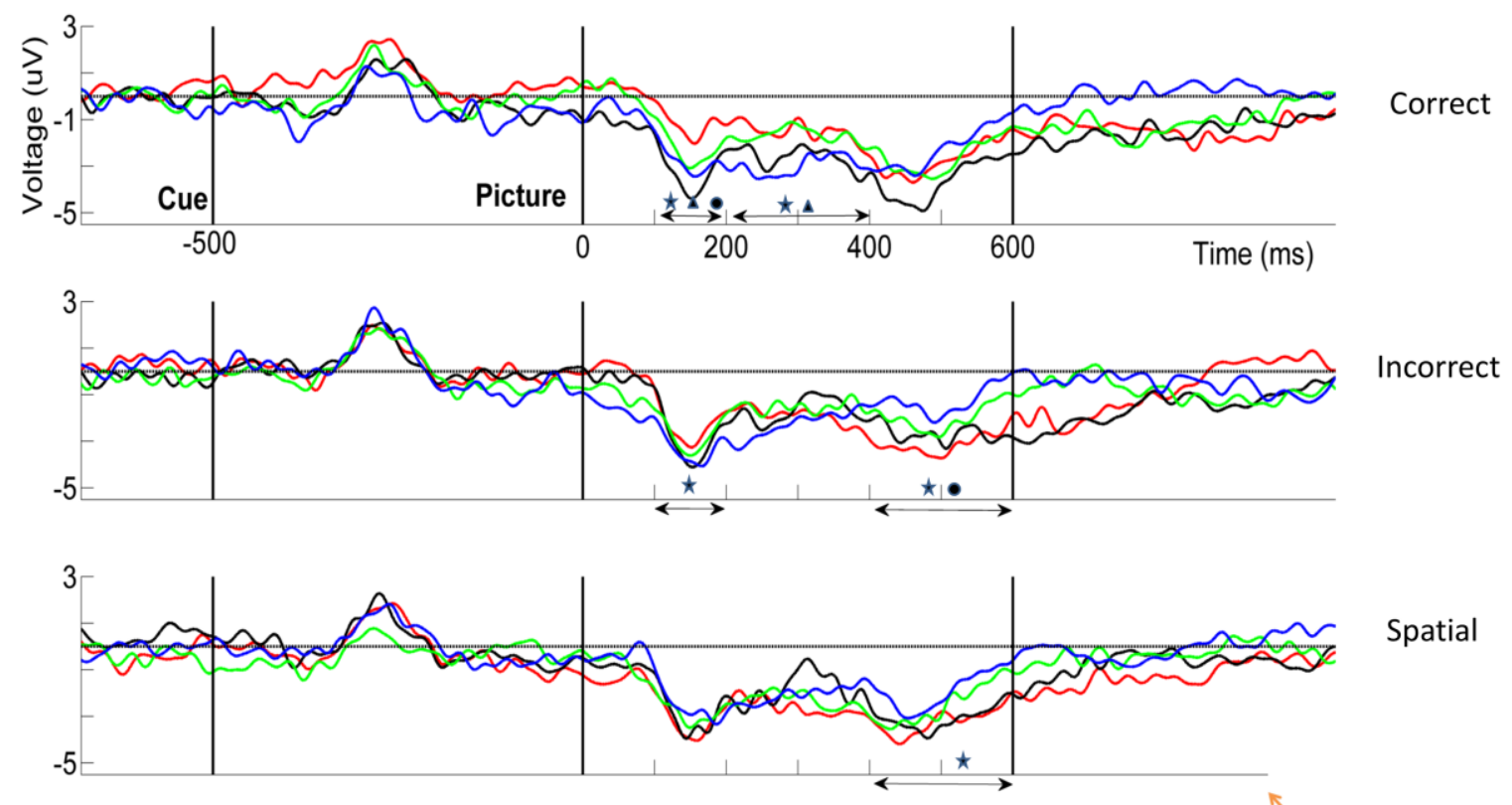

Spatial

B
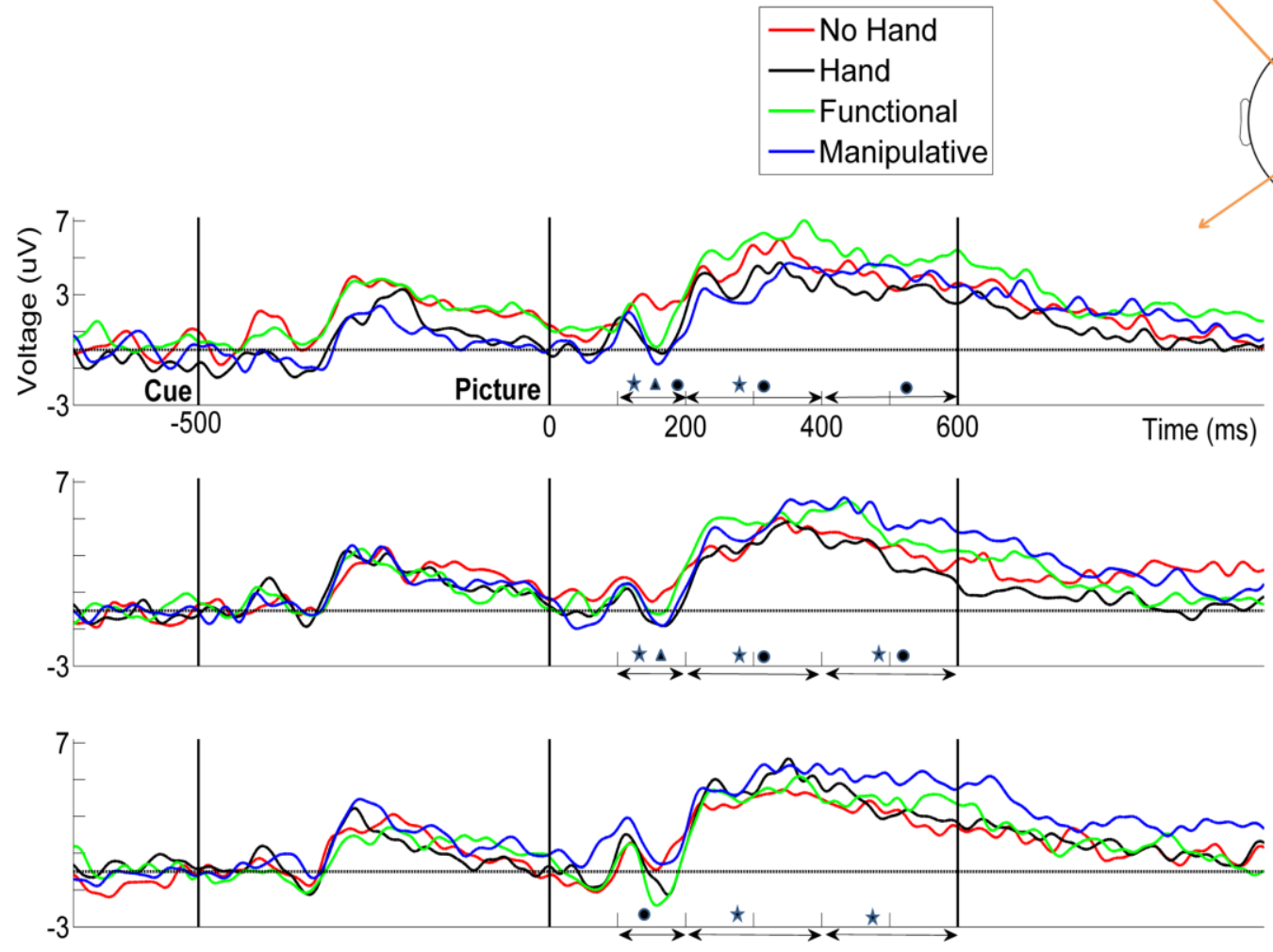
$\underline{A}$

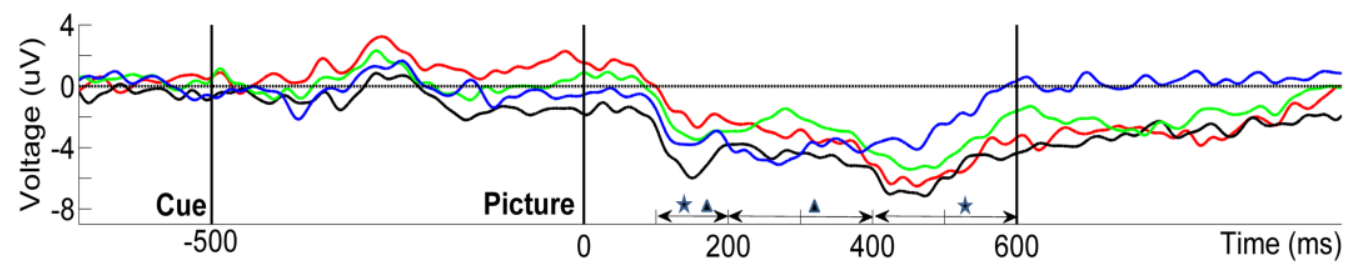

Incorrect
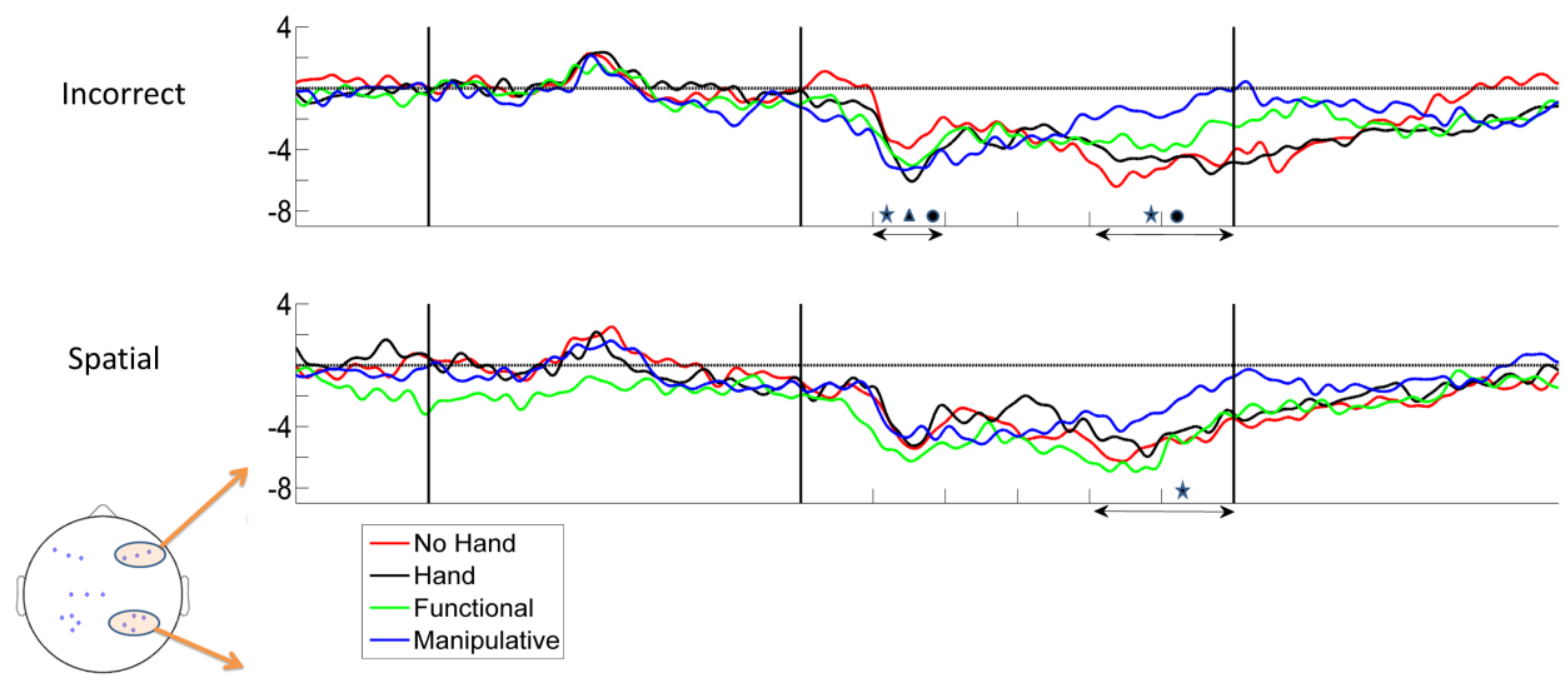

B
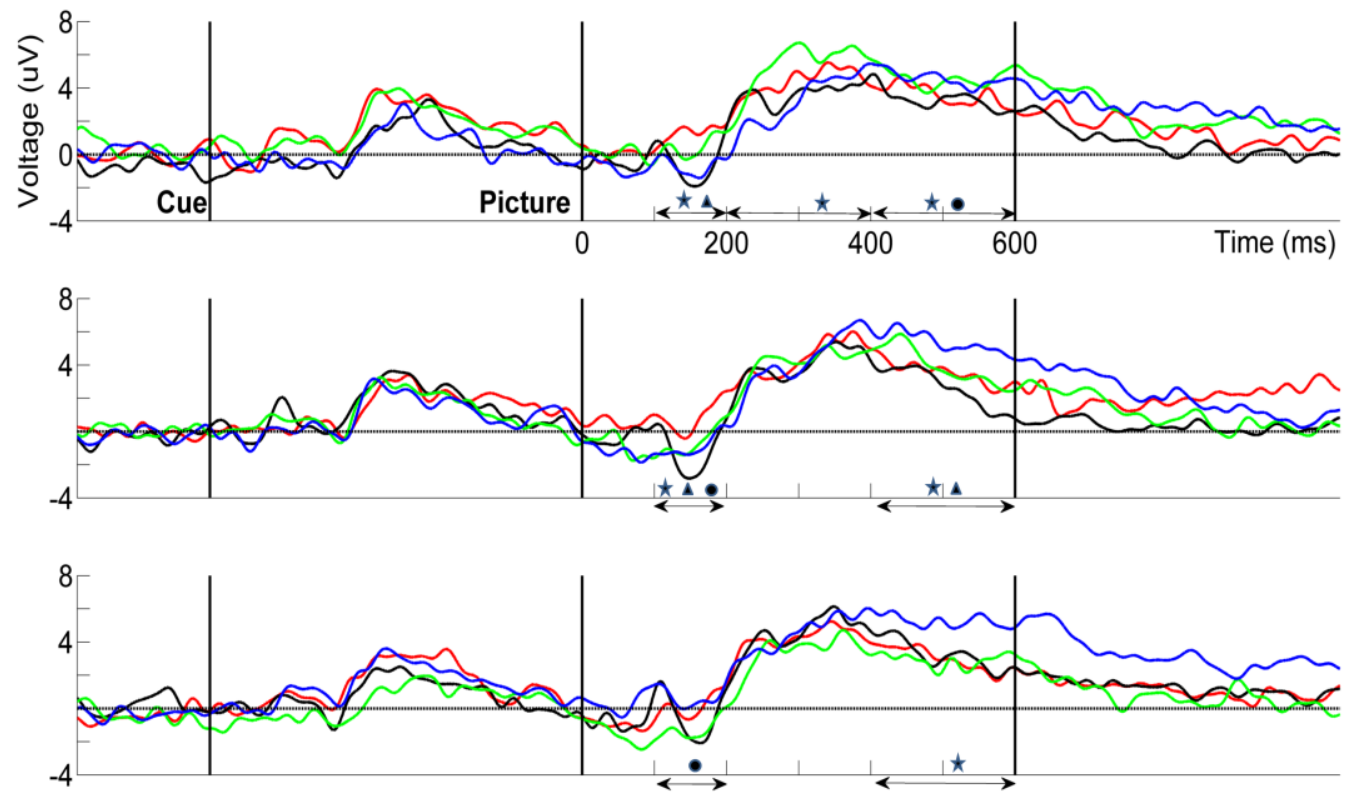


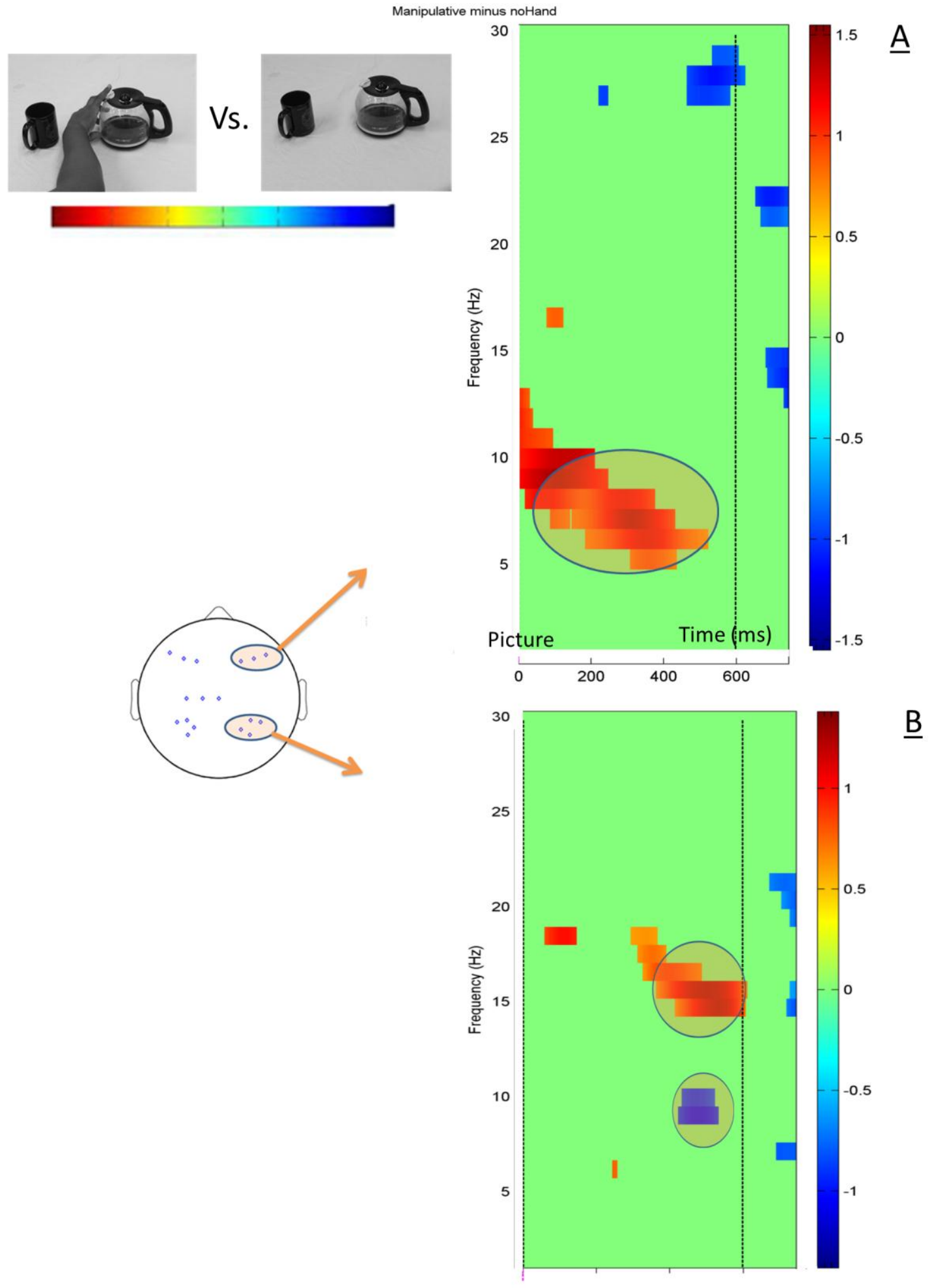




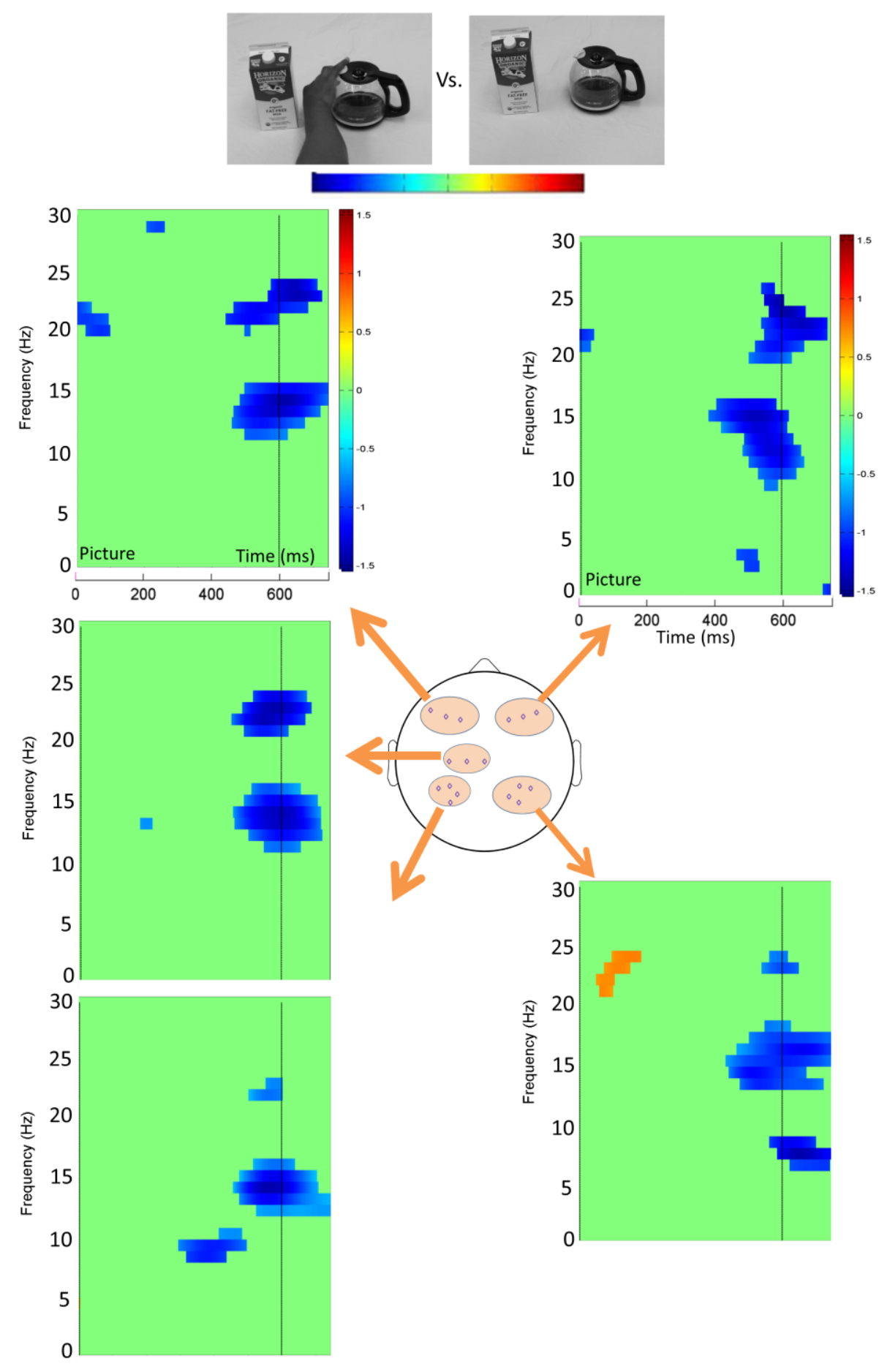

